7. Alderman, W.M., Madhavan, S., Ooi, W.L. et al. Association of the renin-sodium profile with the risk of myocardial infarction in patients with hypertension. $N$ Engl J Med 1991, 324: $1098-1104$.

\section{An unusual case of dysphagia}

Sir,

Regarding the paper of Treasure and Hall, ${ }^{1}$ we wish to report a clinical story of similar symptoms but with a different aetiology.

A 68 year old woman had progressive dysphagia that developed over a period of several months. She had the feeling of food remaining stuck in the upper third of her chest. There were no symptoms of heartburn or other chest distress and no weight loss.

Clinical examination and routine chest $\mathrm{X}$-rays were normal. Barium swallow revealed an oesophageal stenosis and endoscopy demonstrated a pulsatory mass at the $25 \mathrm{~cm}$ level. There were no mucosal abnormalities. A computed tomographic scan with the application of an intravenous contrast medium showed an aberrant right subclavian artery (ARSA) or arteria lusoria, running vertically and behind the oesophagus and causing the stenosis. In order to demonstrate that the dysphagia in the patient was caused by the presence of the ARSA, an evaluation of deglutition with solid food was performed. The food was labelled with technetium-99 in a colloidal suspension and revealed a stagnation for solids at the level of the ARSA, confirming the diagnosis of dysphagia lusoria. The patient was treated by simple division of the ARSA with decompression of the oesophagus. A reanastomosis was not performed since at operation the blood flow to the right arm through collateral circulation was noted to be satisfactory. After the operation the woman was free of any difficulties in swallowing.

Dysphagia lusoria, the symptomatic compression of the oesophagus by the ARSA, is a rare syndrome. ${ }^{2,3}$ The anomalous vessel represents the persisting portion of the right fourth aortic arch, the cranial portion of this arch being obliterated. ${ }^{4}$ Symptoms can appear in childhood and in adults. ${ }^{25.6} \mathrm{~A}$ universally accepted explanation for the absence of clinical signs in children and young adults does not exist. Loss of mobility of the anatomical structures and a subsequent oesophageal motility disorder have been suggested. Furthermore, the dilatation, the tortuosity and rigidity of the great vessels, the increase in the dorsal kyphosis, the sclerosis and calcification of trachea, aorta and the abnormal vessels could be an explanation for the occurrence of dysphagia. ${ }^{2,6}$

\section{Elewaut \\ R. Rubens \\ Department of Endocrinology, University of Gent De Pintelaan 185 9000 Gent, Belgium.}

\section{References}

1. Treasure, T. \& Hall, R.J. An unusual cause of dysphagia: a vascular ring. Postgrad Med J 1994, 70: 648-650.
2. Balaji, M.R., Ona, F.V., Cheeran, D. et al. Dysphagia lusoria: a case report and review of diagnosis and treatment in adults. Am J Gastroenterol 1982, 77: 899-901.

3. Bayford, D. An account of singular case of obstructed deglutition. Mem Med Soc Lond 1794, 2: 275-286.

4. Seres, M., Maros, T. \& Seres, L. The aberrant retroesophageal right subclavian artery. Morphol Embryol 1985, 31: 183-186.

5. Klinkhamer, A.C. The aberrant right subclavian artery. In: Esophography in Anomalies of the Aortic Arch System. Excerpta Medica Foundation, Amsterdam, 1969, pp. 16-30.

6. Pomè, G., Vitali, E., Mantovani, A. et al. Surgical treatment of the aberrant retroesophageal right subclavian artery in adults (dysphagia lusoria). J Cardiovasc Surg 1987, 28: 405-412.

\section{Polycythaemia rubra vera presenting with severe glossitis}

Sir,

Polycythaemia rubra vera (PRV) can present in various ways including acute bleeding or thrombotic episodes, cerebral circulatory disturbances, gout and pruritus. We report an unusual case of PRV in whom the main presenting complaint was severe glossitis.

An 84 year old woman was admitted to hospital with a 3 month history of severe soreness of the tongue with associated loss of taste, prominent dysphagia for solids and weight loss of $12 \mathrm{~kg}$. There was a past history of ischaemic heart disease and hypertension, and a mild stroke with very good recovery 6 years prior to the present admission. At the time of her stroke, routine tests including a full blood count were normal. She had noo history of abdominal or respiratory disease, was a non-smoker and did not drink alcohol. Her only regular medication prior to admission was multivitamin tablets for a few weeks, but the glossitis had not improved.

On examination there was facial plethora, a smooth atrophic, 'beefy' red tongue and moderate splenomegaly. Investigations revealed: haemoglobin $17.8 \mathrm{~g} \%$, white cell count $42.2 \times 10^{9} / 1$, platelet count $195 \times 10^{9} / 1$; differential white cell count-neutrophils $58 \%$, lymphocytes $17 \%$, basophils $10 \%$, myelocytes $10 \%$, myeloblasts $2 \%$; MCV $73 \mathrm{fl}, \mathrm{MCH} 24 \mathrm{pg}, \mathrm{MCHC} 33 \mathrm{~g} / \mathrm{dl}$. Blood film showed a leukoerythroblastic picture and the ESR was $1 \mathrm{~mm}$ in the first hour. Serum electrolytes, renal, liver, calcium and thyroid function tests, as well as serum folate and ferritin levels were normal. Chest X-ray and arterial blood gases were also normal. Serum $B_{12}$ was $>1,000 \mathrm{ng} / 1$ (normal 200-900) and leucocyte alkaline phosphatase score 182 (normal 35-100). Bone marrow trephine examination revealed panhyperplasia with the presence of moderate myelofibrosis, and no evidence of iron deficiency or metastatic disease. An abdominal ultrasound confirmed moderate splenomegaly and showed no abnormality of other abdominal viscera. A barium meal and a gastroscopy were normal. On the basis of these findings, a diagnosis of PRV with secondary myelofibrosis was made and busulphan $4 \mathrm{mg}$ daily was commenced. By the end of 4 weeks' therapy the glossitis and dysphagia resolved, her weight steadily increased, and the full blood count normalized.

Glossitis could occur in PRV as a result of severe iron deficiency due to repeated bleeding or venesections with no iron replacement. ${ }^{1.2}$ However, in our patient the existence of glossitis without iron or vitamin deficiency, and its fast resolution with chemotherapy given for the 
myeloproliferative disease, strongly suggest that it was caused by the underlying condition. Furthermore, to the best or our knowledge, PRV presenting as disabling glossitis has not previously been reported.

M.V.R. Prasad

B. Panayiotou

R. Siddiqui

M.N. Zaman

Department of Geriatrics,

South Wing,

Manor Hospital,

Walsall WS2 9PS, UK.

\section{References}

1. Goldhaber, P. Oral manifestations of disease. In: Petersdorf, R.G., Adams, R.D., Braunwald, E., Isselbacher, K.J., Martin, J.B. \& Wilson, J.D. (eds) Harrison's Principles of Internal Medicine, McGraw-Hill Book Company, New York, 1985, pp. 182-188.

2. Berk, P.D. Polycythaemia vera. In: Andreoli, T.E., Mandell, G.L., Murray, J.F., Nathan, D.G., Ockner, R.K. \& Smith, T.W. (eds) Cecil Textbook of Medicine, 19th edn. W.B. Saunders Company, Philadelphia, 1992, pp. 925-929.

\section{A case of recurrent eclampsia}

Sir,

Eclampsia occurring in successive pregnancies, especially after the second, is a relatively rare condition. ${ }^{1.2}$ We describe here a case who presented with recurrent eclampsia in all her five pregnancies.

A 25 year old gravida 5 came to us at 34 weeks gestation with over 20 generalized tonic-clonic convulsions. She had complained of headache preceding the fits; there was no previous history of fever, vomiting, epigastric pain or blurring of vision. She had not had any antenatal check ups. The woman was not an epileptic but had developed similar tonic-clonic convulsions intranatally in each of her four previous pregnancies. The first child was stillborn at term. The second and fourth children, both daughters, 6 years and 1 year old now are growing well. The third child born at 32 weeks gestation died after 10 days. She had a normal delivery each time following augmentation of labour and recovered rapidly after delivery, becoming normotensive without medication at discharge. She had not come for antenatal visits in any of the pregnancies and did not report for follow-up despite adequate counselling. The entire family was illiterate.

At admission, she was semicomatose, the pulse rate $120 /$ minute, the blood pressure $210 / 130 \mathrm{mmHg}$, tempera- ture $102^{\circ} \mathrm{F}$, there was no peripheral oedema. The uterus was 34 weeks in size, the presentation cephalic and the head not engaged. The uterus was contracting mildly, once every 10 minutes, lasting 20 seconds and the cervical score was 5 .

An intravenous diazepam infusion, phenytoin sodium (100 mg every 8 hours) and nifedipine (10 mg sublingually immediately and every 6 hours), was started along with life support measures. Low rupture of membranes was carried out and oxytocin augmentation started. The woman delivered a live $1.6 \mathrm{~kg}$ female baby within 2 hours vaginally (Apgar 6/10 at 1 minute and $8 / 10$ at 5 minutes). The baby is now growing well. The PCV was $34 \%$, urinary albumin present in traces, serum urea creatinine uric acid and liver enzymes were within the normal range, as were the fibrinogen and fibrin degradation product levels. The platelet count was $\left(120 \times 10^{9} / 1\right)$, the ocular fundi were normal.

The patient recovered well following delivery. There were no further convulsions and her blood pressure was $130 / 80 \mathrm{mmHg}$ by the fourth postpartum day without medication. She was discharged on the seventh postpartum day, but despite intensive counselling was again not willing for any form of contraception and failed to report for any subsequent follow-up.

Chesley et al. ${ }^{1}$ have reported that $2 \%$ of primiparous eclamptics have recurrent eclampsia in the second pregnancy and Sibai and coworkers ${ }^{3}$ have also reported a $1.4 \%$ incidence of eclampsia in the second pregnancies of such women. We, however, did not come across any report of eclampsia occurring in each of five pregnancies of any women. Home visits with more counselling at home appear necessary for this patient to prevent any future recurrence.

M. Misra

S. Mishra

Department of Obstetrics and Gynaecology Jawaharlal Nehru Hospital and Research Centre, Bhilai-490 006 (MP), India.

\section{References}

1. Chesley, L.C., Annitto, J.E. \& Cosgrove, R.A. Prognostic significance of recurrent toxaemia of pregnancy. Obste Gynecol 1964, 23: 874-881.

2. Chesley, L.C., Annitto, J.E. \& Cosgrove, R.A. Remote prognosis of eclamptic women: sixth periodic report. Am J Obstet Gynecol 1976, 124: 446-459.

3. Sibai, B.M., El Nazar, A. \& Gonzaliz Ruiz, A. Severe pre-eclampsia-eclampsia in young primigravid women: subsequent pregnancy outcome and remote prognosis. Am J Obstet Gynecol 1986, C155: 1011-1016. 